Herzschr Elektrophys 2015 $26: 3-4$ DOI 10.1007/s00399-015-0356-8

Online publiziert: 18. Februar 2015

(c) Springer-Verlag Berlin Heidelberg 2015

\section{Hendrik Bonnemeier ${ }^{1}$. Jürgen Potratz ${ }^{2}$}

${ }^{1}$ Klinik für Innere Medizin III, Kardiologie, Angiologie, Intensivmedizin, Campus Kiel, Kiel, Deutschland

2 Med. Klinik I, Agaplesion Diakonieklinikum, Rotenburg/Wümme, Deutschland

\title{
Risikostratifikation des plötzlichen Herztodes
}

Sehr geehrte Leserinnen, sehr geehrte Leser,

das vorliegende Sonderheft der Zeitschrift Herzschrittmachertherapie + Elektrophysiologie widmet sich dem Thema "Risikostratifikation “. Die Prädiktion des plötzlichen Herztodes (PHT) ist und bleibt eine der größten Herausforderungen in der modernen Kardiologie. Mit einer jährlichen Inzidenz von etwa 1 pro 1000 Einwohner ist der PHT eine sehr häufige und klinisch relevante Entität. Seit über einer Dekade hat sich die Therapie mit implantierbaren Kardioverter-Defibrillatoren (ICD) als ein effektives Verfahren zur Verhinderung des PHT etabliert. Nach der Veröffentlichung der MADIT-II-Studie und der SCD-HeFT-Studie ist die Zahl der ICD-Implantationen zur Primärprophylaxe des PHT exponentiell angestiegen. In diesen Studien wurde als primärer Risikoprädiktor eine linksventrikuläre Ejektionsfraktion $<30 \%$ (MADIT-II) bzw. $<35 \%$ (SCD-HeFT) verwendet. Die genannten Studien konnten einen signifikanten Überlebensvorteil in der ICDPopulation zeigen. Allerdings muss man festhalten, dass die mittlere linksventrikuläre Ejektionsfraktion (LVEF) der Patienten der MADIT-II-Studie $23 \%$ und die der SCD-HeFT-Studie $25 \%$ betrug - die Standardabweichung der mittleren Ejektionsfraktion in beiden Studien erreichte nicht einmal 30 bzw. $35 \%$. Somit hat einer der renommiertesten Wissenschaftler auf dem Gebiet der Risikoprädiktion des PHT, Robert J. Myerburg, Recht mit der Feststellung ,it is the entry criterion and not the group actually studied that has driven practice guidelines". In der Tat ist die LVEF derzeit der gebräuchlichste und somit wichtigste klinische Risikoprädiktor für den PHT. Allerdings hat die LVEF eine niedrige Spe- zifität - mehr als $50 \%$ aller PHT-Patienten weisen eine LVEF > $35 \%$ auf. In der sekundärprophylaktischen CASH-Studie wiesen Patienten nach einem überlebten PHT eine mittlere LVEF von 46\% auf. Ferner variiert die LVEF in Bezug auf das Untersuchungsverfahren (Echokardiographie, 2-D-Echokardiographie, Magnetresonanztomographie, Computertomographie, Myokardszintigraphie etc.) und weist bekanntermaßen eine hohe Intra- und Interobserver-Variabilität auf. Fakt ist, dass man nach den MADIT-IIund SCD-HeFT-Kriterien 15 bis $20 \mathrm{~Pa}$ tienten mit einem ICD-versorgen muss, um einem Patienten das Leben zu retten. Fakt ist, dass eine Vielzahl von Patienten, welche aktuell primärpräventiv mit einem ICD versorgt werden, eine LVEF zwischen 30 und $35 \%$ aufweisen - also nicht im engeren Sinne den Studienpatienten von MADIT-II und SCD-HeFT entsprechen. Und Fakt ist auch, dass die Anzahl inadäquater Schockauslösungen sowohl in Studien als auch im klinischen Alltag innerhalb der ersten Jahre nach primärprophylaktischer ICD-Implantation deutlich über der Anzahl adäquater Schockauslösungen liegt, und inadäquate Schockauslösungen (bei supraventrikulären Tachykardien oder Elektrodenproblemen) mit einer erhöhten Mortalitätswahrscheinlichkeit einhergehen. Somit kann heute sicher festgestellt werden, dass mit der alleinigen Ermittlung der LVEF noch lange nicht der heilige Gral der Risikostratifikation gefunden worden ist.

Viele vorangegangene Studien konnten eindrucksvoll demonstrieren, dass eine Kombination aus funktionellen (LVEF) und elektrophysiologischen Parametern sowohl die Sensitivität als auch die Spezifität bzgl. der Prädiktion des PHT erhöhen können. In der MADIT-I- und in der MUST-Studie liegt bei Patienten mit reduzierter LVEF und in der elektrophysiologischen Untersuchung (EPU) induzierbarer Kammertachykardie nach ICD-Implantation die ,number needed to treat" (NNT) unter 4 Patienten und 1 Leben zu retten. Auch Ergebnisse aus Studien an Herzinsuffizienzpatienten, die mittels Parametern des kardialen autonomen Nervensystems (Herzfrequenzvariabilität, Baroreflex-Sensititvität, Herzfrequenzturbulenz, Dezelerationskapazität etc.), QT-Intervall-Variabilität, oder T-Wellen-Alternans untersucht worden sind, lassen den Rückschluss zu, dass eine Kombination von Risikoparametern eine deutlich genauere Risikostratifikation zulässt. Wichtig ist in diesem Zusammenhang vor allem die Unterscheidung der pathophysiologischen Ursachen der zum PHT führenden Herzrhythmusstörung. Sind es bei Patienten mit einer ischämischen Kardiomyopathie (ICMP) in der Regel Makro-Reentrymechanismen durch ein infarktinduziertes Substrat, also häufig monomorphe Kammertachykardien, welche im Verlauf in ein Kammerflimmern degenerieren, so sind bei Patienten mit einer nichtischämischen dilatativen Kardiomyopathie (DCMP) eher polymorphe Kammertachykardien und primäres Kammerflimmern die Ursache für den PHT. Somit sollte eine moderne Risikostratifikation möglichst zwischen beiden Entitäten differenzieren.

In der aktuellen Ausgabe von Herzschrittmachertherapie + Elektrophysiologie soll deshalb erstmals systematisch zwischen der Risikostratifikation bei Patienten mit ICMP und DCMP differenziert werden. Ein ganz herzlicher Dank gilt dem Verlag und dem Herausgeber, welche den national und international renommierten Autoren die Möglichkeit ge- 


\section{Editorial}

geben haben, die unterschiedlichen Parameter und Untersuchungstechniken der Risikostratifikation bei Patienten mit ICMP und DCMP zu beleuchten. Ein besonderer Dank gilt auch den Autoren, die klar und klinisch nachvollziehbar die Thematik für den Leser dargelegt haben. Vielleicht trägt dieses Heft dazu bei, dass zukünftig die Indikation zur primärprophylaktischen ICD-Implantation ein wenig differenzierter gestellt werden kann vielleicht benötigen einige Ihrer Patienten - liebe Leserinnen und Leser - mit einer LVEF > 35\% eher einen ICD als andere Patienten mit einer LVEF um $30 \%$.

Wir wünschen Ihnen eine umfassende, informative und gewinnbringende Lektüre.

Mit herzlichen Grüßen,

Ihr Hendrik Bonnemeier und Ihr Jürgen Potratz

\section{Korrespondenzadresse}

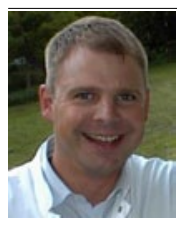

Prof. Dr. H. Bonnemeier

Klinik für Innere Medizin III

Kardiologie, Angiologie

Intensivmedizin, Campus Kiel

Schittenhelmstr. 12, 24105 Kiel

Hendrik.Bonnemeier@uk-sh.de 